\title{
PALINOLOGÍA DE ANGIOSPERMAS DE LA FORMACIÓN MONTE CHICO (CRETÁCICO SUPERIOR) DE LA PROVINCIA DE SANTA CRUZ, ARGENTINA
}

\author{
LETICIA POVILAUSKAS \\ División Paleobotánica, Museo Argentino de Ciencias Naturales "Bernardino Rivadavia", CONICET, \\ Av. Ángel Gallardo 470, C1405DJR, Buenos Aires, Argentina.lepovilauskas@macn.gov.ar
}

\begin{abstract}
ANGIOSPERMS PALYNOLOGY FROM MONTE CHICO FORMATION (UPPER CRETACEOUS) OF SANTA CRUZ PROVINCE, ARGENTINA. Diverse palynological assemblages composed by both continental and marine palynomorphs were recovered from the Monte Chico Formation, Upper Cretaceous, Santa Cruz Province. In this contribution palynological assemblages were studied, and the samples were taken from the entire section of this unit. Fifty five species of angiosperm pollen grains were determined. They belong to the genera: Arecipites, Beaupreaidites, Clavamonocolpites, Clavatricolpites, Ericipites, Forcipites, Haloragacidites, Ilexpollenites, Liliacidites, Longapertites, Nothofagidites, Peninsulapollis, Periporopollenites, Proteacidites, Pseudowinterapollis, Psilatricolpites, Psilatricolporites, Quadraplanus, Rhoipites, Rousea, Senipites, Sparganiaceapollenites, Spinizonocolpites, Triatriopollenites, Tricolpites, Tricolporites and Triporopollenites. On the basis of the stratigraphic distribution of the identified species, mainly in Argentina and Antarctica, and together with the preliminary analysis of spores, a Maastrichtian-Late Maastrichtian age is here suggested. The palynological assemblages obtained would typically indicate a near shore marine environment of deposition, with marginal conditions towards to the upper section.
\end{abstract}

Key words: Angiosperms, Monte Chico Formation, Cretaceous, Argentina.

RESUMO - Diversas associações palinológicas foram recuperadas dos sedimentos da Formação Monte Chico, Cretáceo Superior, Província de Santa Cruz, sendo constituidas por palinomorfos marinhos e continentais. As amostras são provenientes da parte superior desta unidade. Nesta contribuição são apresentadas 55 espécies de grãos de pólen identificadas como pertencentes aos seguintes gêneros de angiospermas: Arecipites, Beaupreaidites, Clavamonocolpites, Clavatricolpites, Ericipites, Forcipites, Haloragacidites, Ilexpollenites, Liliacidites, Longapertites, Nothofagidites, Peninsulapollis, Periporopollenites, Proteacidites, Pseudowinterapollis, Psilatricolpites, Psilatricolporites, Quadraplanus, Rhoipites, Rousea, Senipites, Sparganiaceapollenites, Spinizonocolpites, Triatriopollenites, Tricolpites, Tricolporites e Triporopollenites. Com base na distribuição estratigráfica das espécies identificadas, principalmente na Argentina e na Antártida e, em conjunto com a análise preliminar dos esporos, sugere-se a idade Maastrichtiano-Neomaastrichtiano. A assembleia palinológica indica um ambiente deposicional tipicamente litorâneo, com continentalização progressiva para a parte superior da seção.

Palavras-chave: Angiospermas, Formação Chico Monte, Cretáceo, Argentina.

\section{INTRODUCCIÓN}

En esta contribución se continúa con el estudio sistemático de los palinomorfos de la Formación Monte Chico, Maastrichtiano de la Provincia de Santa Cruz, Argentina, obtenidos de secciones aflorantes en dos zonas de estudio, próximo al límite con Chile. En las dos contribuciones (Povilauskas, 2011, 2012) anteriores se describieron sistemáticamente las esporas de pteridófitas y briófitas, y el polen de gimnospermas, junto al marco geológico, mapa de ubicación (Figura 1), señalando las dos zonas de estudio (Estancia San José y Estancia Laguna Salada), y el perfil estratigráfico con los niveles de muestreo (Figura 2). En el presente trabajo se describen todos los granos de polen pertenecientes a las angiospermas presentes, incluidos aquellos con ubicación sistemática incierta y especies asignadas al cf. en dicha unidad, que permiten realizar inferencias paleoclimáticas y paleoambientales para esta formación. Además, se ilustraron todas las especies presentes en la Formación Monte Chico.

\section{MATERIAL Y MÉTODOS}

Las muestras han sido colectadas en las zonas que presentan las mejores exposiciones llegando a un total de 36 niveles muestreados entre las dos zonas de estudio (Estancia San José y Estancia Laguna Salada) (Figura 2). El distanciamiento entre muestras fue irregular, dependiendo de la litología y de los tramos cubiertos. En intervalos convenientes el muestreo se efectuó a un distanciamiento de 5 


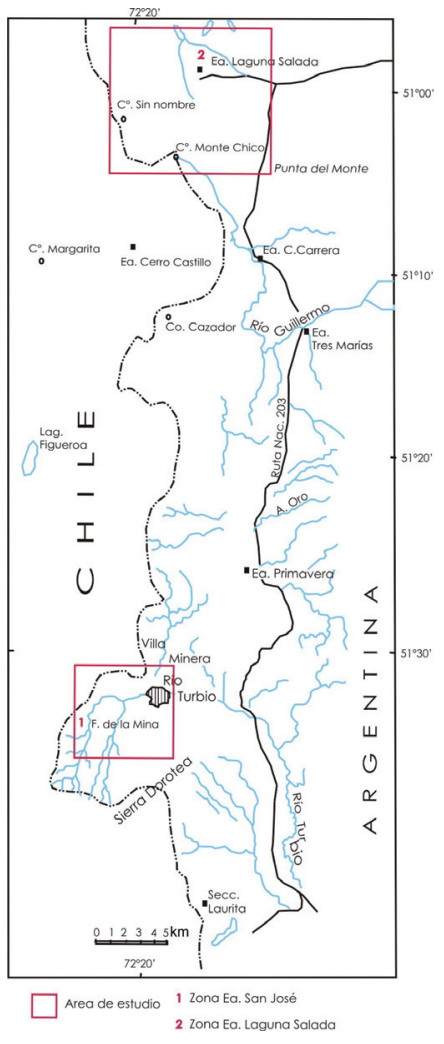

Figura 1. Mapa de ubicación geográfica.

Figure 1. Geographic location map.

$\mathrm{m}$. El material recolectado fue embolsado y convenientemente rotulados para su posterior análisis en el laboratorio.

La extracción palinológica se realizó siguiendo métodos convencionales de extracción física y química para palinología (HCl-HF) y al residuo obtenido se lo filtró mediante mallas de $(+10$ y +25 micrones). Las preparaciones definitivas fueron montadas en gelatina glicerina. Los ejemplares fueron estudiados con el microscopio Leitz Wetzlar Germany y Olympus BX51 de la Sección de Paleopalinología y fueron fotografiados con el microscopio óptico de transparencia y el electrónico de barrido (MEB), del Museo Argentino de Ciencias Naturales "Bernardino Rivadavia". Para la documentación fotográfica de los ejemplares se utilizó una cámara digital Nikon E4500. Se consideró el sistema de clasificación semi-natural (Pocock, 1962) con la utilización de morfotaxones a nivel de género y especie.

El material estudiado se encuentra depositado en la Colección Palinológica del Museo Regional Provincial "Padre Manuel Jesús Molina" de la ciudad de Río Gallegos, Provincia de Santa Cruz, Argentina bajo los números de catálogo 1943 al 1978.

En los diagramas de frecuencias relativas (Figuras 3, 4; Tablas 1,2) sólo se consideraron las muestras más fértiles desde el punto de vista palinológico de toda la secuencia analizada, con el fin de obtener datos más representativos: 1945, 1947, 1949, 1952-1957, 1959 y 1964.

Abreviaturas. MPM-MP, Museo Regional Provincial "Padre Manuel Jesús Molina”, colección Río Gallegos, Provincia de Santa Cruz, Argentina; DE, diámetro ecuatorial; DP, diámetro polar.

\section{PALEONTOLOGÍA SISTEMÁTICA DE TAXA SELECCIONADAS}

Anteturma POLLENITES Potonié, 1931

Turma PLICATES Naumova emend. Potonié, 1960

Subturma TRIPTYCHES Naumova, 1939

Beaupreaidites (Cookson) Martin, 1973

Especie tipo. Beaupreaidites elegansiformis (Cookson, 1950) Couper, 1953.

\section{Beaupreaidites sp.1 \\ (Figura 5C)}

Descripción. Grano de polen tricolpado, de contorno subtriangular en vista polar, lados convexos y bordes angulosos. Colpos cortos, formados por un adelgazamiento de la nexina. Exina gruesa, de $2-3 \mu \mathrm{m}$ de espesor, microperforada, semitectada.

Dimensiones. (3 especímenes medidos) DE 29(31,5)33 $\mu \mathrm{m}$. Material. MPM-MP 1955 99/15,4; MPM-MP 1973 89/5.

Procedencia geográfica. Formación Monte Chico, Cuenca Austral, Provincia de Santa Cruz, Argentina.

Observaciones. La especie aquí descripta se aparta de Beaupreaidites elegansiformis (Cookson, 1950), por presentar dimensiones menores y una exina algo más gruesa y microperforada, no observándose un tectum contínuo.

Afinidad botánica. Familia Proteaceae.

Turma PLICATI (Naumova, 1937-1939) Potonié, 1960

Subturma MONOCOLPATES (MONOSULCITES y ZONOCOLPATES) Potonié, 1970

Infraturma SCULPTATI Potonié, 1970

\section{Clavamonocolpites Gonzalez, 1967} emend. Muller et al., 1987

Especie tipo. Clavamonocolpites terrificus Guzmán, 1967.

\section{Clavamonocolpites sp.1}

(Figuras 5D,E)

Descripción. Grano de polen monocolpado, anisopolar, bilateral, de contorno elíptico y bordes redondeados. Colpo extendido hasta los bordes del grano, amplio. Exina tectada de 1,5 $\mu \mathrm{m}$ de espesor; esculturada con clavas. Clavas largas, distribuidas irregularmente, de 3-3,5 $\mu \mathrm{m}$ de largo y 1-1,5 $\mu \mathrm{m}$ de ancho. Los elementos esculturales se disponen de manera apretada sobre la superficie del grano. Nexina de 0,5-1 $\mu \mathrm{m}$ de espesor.

Dimensiones. (1 espécimen medido) DP $32 \mu \mathrm{m}$; DE $24 \mu \mathrm{m}$. Material. MPM-MP 1947 91,4/6; MPM-MP 1971 112,5/5,7; MPM-MP 1968 103,5/9,6.

Procedencia geográfica. Formación Monte Chico, Cuenca Austral, Provincia de Santa Cruz, Argentina.

Observaciones. Esta especie se registra esporádicamente en la Formación Monte Chico Clavamonocolpites sp. 1 descripta 
Litología

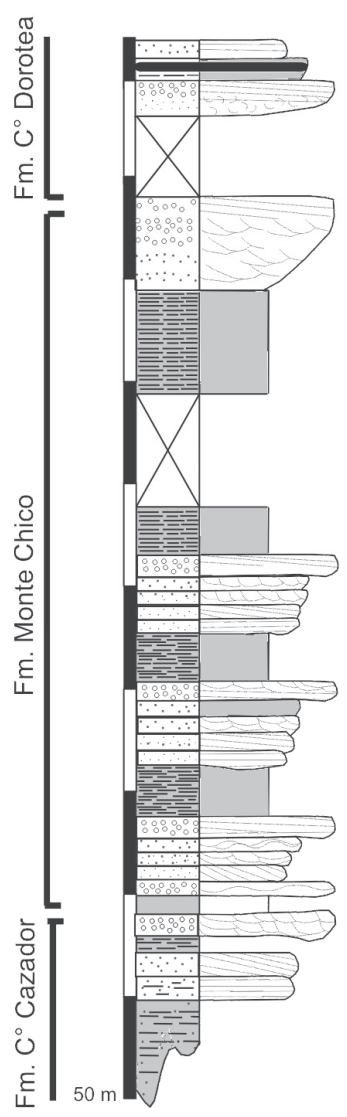

MPM-MP 1978

MPM-MP 1977

MPM-MP 1976

MPM-MP 1975

MPM-MP 1974

MPM-MP 1973

MPM-MP 1972

MPM-MP 1971

MPM 1969

MPM-MP 1967-1968

MPMP $1905-1066$

MPM-MP 1963-1964

MPM-MP 1963-1964

MPM-MP 1961-1962

MPM-MP 1957-195

MPM-MP 1955-195

MPM-MP 1953-1954

MPM-MP 1951-1952

MPM-MP 1949-1950

MPM-MP 1947-1943

MPM-MP 1945-1948

MPM-MP 1943-1944

Figura 2. Perfil de la Formación Monte Chico.

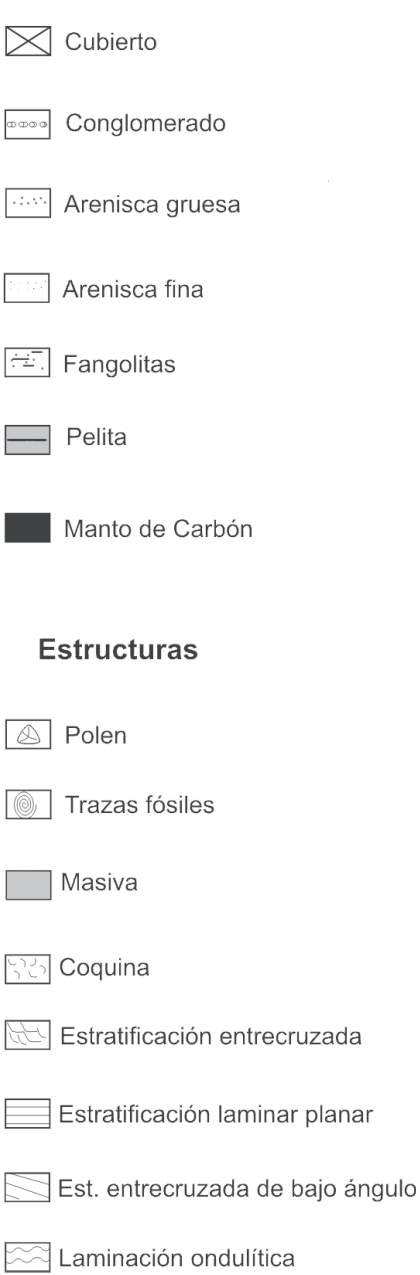

Figure 2. Stratigraphic section of the Monte Chico Formation.

por Jaramillo \& Dilcher (2001) para el Paleógeno Medio de Colombia, presenta dimensiones mayores y la esculturación está compuesta por clavas más cortas $(2 \mu \mathrm{m})$.

Afinidad botánica. Familia Palmae.

Subturma TRICOLPATES, TRIPTYCHES Naumova, 1939 Infraturma ISOTRICOLPATI Potonié, 1970

\section{Clavatricolpites Pierce, 1961}

Especie tipo. Clavatricolpites densiclavatus Pierce, 1961.

\section{Clavatricolpites sp. 1}

(Figuras 5F-H)

Descripción. Grano de polen tricolpado, isopolar, de simetría radial, prolado y contorno circular. Colpo extendido hasta el borde del grano, con márgenes irregulares, mal definidos. Ectocolpios simples, membrana colpal generalmente ausente. Exina intectada, nexina $(0,5-1 \mu \mathrm{m}$ de espesor) relativamente fina, sexina $(0,5-0,8 \mu \mathrm{m}$ de espesor) esculturada con clavas de 1-1,5 $\mu \mathrm{m}$ de largo, 0,5-
$1 \mu \mathrm{m}$ de ancho en el tope y menos de $0,5 \mu \mathrm{m}$ de ancho en la base, distribuidas de manera ordenada y regular. Clavas de la exina a veces se encuentran fusionadas formando un pseudoretículo incompleto.

Dimensiones. (3 especímenes medidos) DE 23(27)30 $\mu \mathrm{m}$. Material. MPM-MP 1947 91,4/6; 94/15,4; MPM-MP 1951 98,7/20,3.

Procedencia geográfica. Formación Monte Chico, Cuenca Austral, Provincia de Santa Cruz, Argentina.

Observaciones. Clavatricolpites densiclavatus (Jaramillo \& Dilcher, 2001) es una forma comparable con los especímenes analizados, pero difiere por presentar dimensiones algo mayores (42 $\mu \mathrm{m}$ de diámetro ecuatorial) y las clavas se disponen sobre la exina de manera más densa.

Afinidad botánica. Angiosperma incertae sedis.

Subturma MONOCOLPATES Iversen \& Troles-Smith, 1950 Infraturma SCULPTATI Potonié, 1970

Liliacidites Couper, 1953

Especie tipo. Liliacidites kaitangataensis Couper, 1953. 


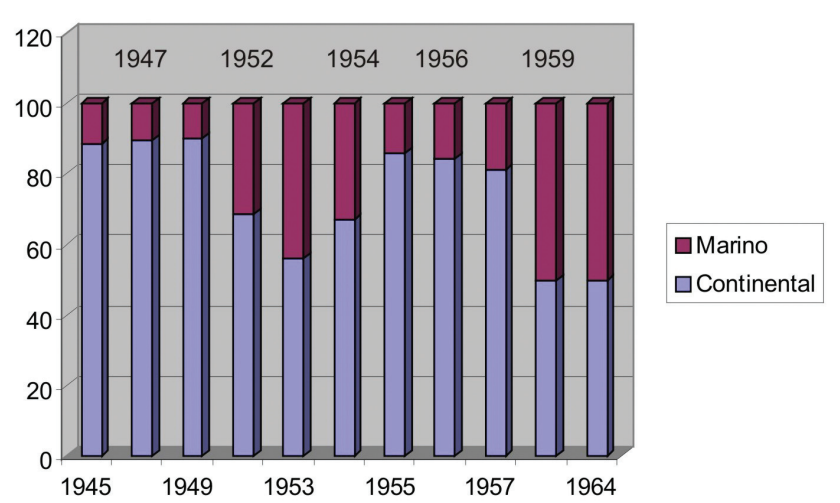

Figura 3. Diagrama de frecuencias relativas (palinomorfos marinos/ continentales), en las dos zonas estudiadas.

Figure 3. Diagram of relative frequencies (palynomorphs marine/ continental) in the two areas studied.

\section{Liliacidites sp. 1}

(Figura 6A)

Descripción. Grano de polen monocolpado, de contorno elíptico a ovalado con extremos redondeados. El colpo se extiende a lo largo de todo el grano, llegando hasta los polos. Exina tectada, de 1,5 $\mu \mathrm{m}$ de espesor, diferenciada en nexina y sexina, reticulada. Retículo heterobrocado, con lúmenes poligonales a subpoligonales, de 0,8-1 $\mu \mathrm{m}$ de diámetro máximo, disminuyendo hacia los polos y colpos, donde presentan menos de $0,5 \mu \mathrm{m}$ de diámetro mínimo. Muros del retículo de 0,3-0,5 $\mu \mathrm{m}$ de ancho, simplibaculados. En algunos especímenes es difícil de observar la esculturación de la exina por el estado de preservación del grano.

Dimensiones. (2 especímenes medidos) DE 22-24 $\mu$ m; DP 32-33,5 $\mu \mathrm{m}$.

Material. MPM-MP 1949 102,1/14,5; MPM-MP 1947 100,8/13,5; 91,5/14,5; 105,6/9,7; MPM-MP 1946 109,6/6.

Procedencia geográfica. Formación Monte Chico, Cuenca Austral, Provincia de Santa Cruz, Argentina.

Observaciones. Liliacidites $\mathrm{sp}$. 1 presenta similitudes con Liliacidites kaitangataensis (Couper, 1953); coincidiendo en su aspecto general, se aparta, sin embargo, por presentar dimensiones algo menores y un retículo con lúmenes más chicos y muros más angostos.

Afinidad botánica. Familia Iridaceae o Liliaceae.

Subturma TRIPTYCHES Naumova, 1939

Peninsulapollis Dettmann \& Jarzen, 1988

Especie tipo. Peninsulapollis gillii Cookson, 1957.

\section{Peninsulapollis sp.1}

(Figura 6K)

Descripción. Grano de polen tricolpado, anguloaperturado, isopolar, oblato a peroblato, de contorno subtriangular con ápices redondeados y lados cóncavos o convexos. Exina de hasta $1,5 \mu \mathrm{m}$ de espesor, estratificada, diferenciada en dos capas no claramente distinguibles. Colpos meridionales de 2,5-4 $\mu \mathrm{m}$ de profundidad, extendiéndose a menos de la mitad de la distancia a los polos. Superficie de la exina escabrada o en algunos casos ornamentada con pequeñas elevaciones y, en menor proporción, microgránulos dispersos irregularmente.

Dimensiones. (2 especímenes medidos) DE 22-26 $\mu \mathrm{m}$.

Material. MPM-MP 1947 100/20,2; MPM-MP 1957 115/14; 92,4/1,7; 85,6/1,4.

Procedencia geográfica. Formación Monte Chico, Cuenca Austral, Provincia de Santa Cruz, Argentina.

Observaciones. Peninsulapollis sp. 1 aparece con regularidad en diferentes niveles a lo largo de las formaciones Cerro Cazador y Monte Chico (Povilauskas, 2010, 2011), y difiere de Peninsulapollis gillii (Cookson) Dettmann \& Jarzen, 1988 por presentar los colpos menos profundos y más estrechos, casi sin dejar lagunas en el ecuador y una esculturación de la exina más delicada. Las dimensiones del grano son coincidentes.

Afinidad botánica. Familia Proteaceae.

Psilatricolpites (van der Hammen, 1956) van der Hammen \& Wijmstra, 1964

Especie tipo. Psilatricolpites clarissimus van der Hammen, 1956.

\section{Psilatricolpites sp.1}

(Figura 6T)

Descripción. Grano de polen tricolpado, escabrado, prolado a perprolado en vista ecuatorial. Colpos profundos y largos que se extienden hasta los polos; sin margo. Exina de $1 \mu \mathrm{m}$ de espesor, psilada a escabrada.

Dimensiones. (4 especímenes medidos) DE 26(28)30 $\mu$ m; DP 27(38) $45 \mu \mathrm{m}$.

Material. MPM-MP 1946 37/95,6; 95,6/5; MPM-MP 1957 97/11; 112,5/20; MPM-MP 1960 89/6,4.

Procedencia geográfica. Formación Monte Chico, Cuenca Austral, Provincia de Santa Cruz, Argentina.

Observaciones. Los especímenes estudiados difieren de la especie Psilatricolpites patagonicus Freile (1972), por presentar un tamaño ligeramente mayor y una exina psilada a escabrada; el resto de las características son concordantes. Psilatricolpites sp. 1 se presenta con frecuencia en los niveles inferiores y medios de la Formación Monte Chico.

Afinidad botánica. Angiosperma incertae sedis. Subturma PTYCHOTRIPORINES Naumova, 1939
Infraturma PROLATI Erdtman, 1943

Psilatricolporites Van der Hammen, 1956

Especie tipo. Psilatricolporites inornatus (Van der Hammen) Pierce, 1961.

Psilatricolporites $\mathrm{sp} .1$

(Figura 7B) 
Nivel 1945

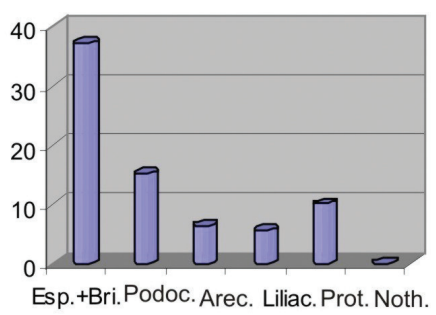

Nivel 1947

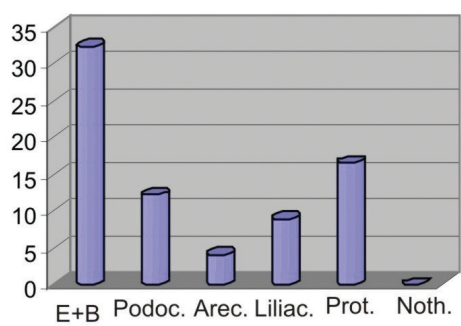

Nivel 1949

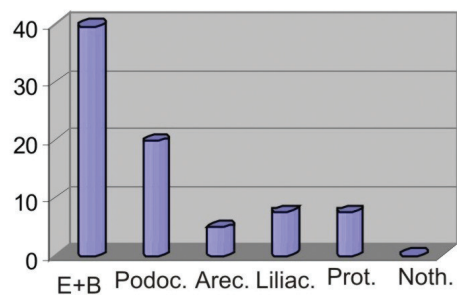

Nivel 1953

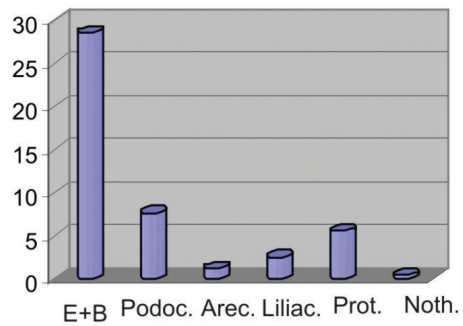

Nivel 1952

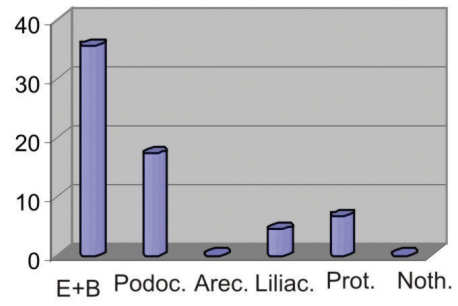

Nivel 1954

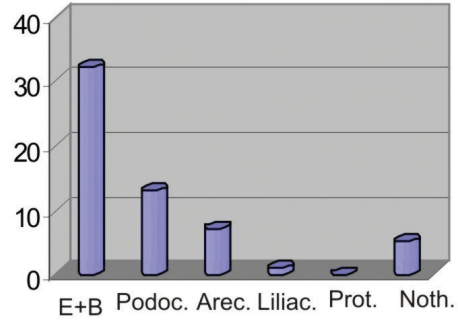

Nivel 1955

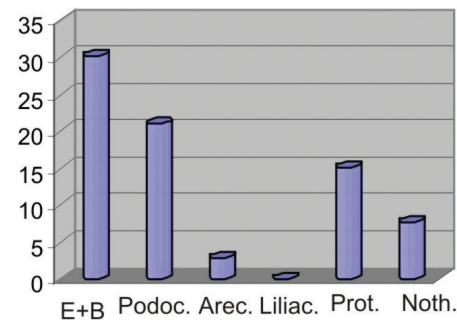

Nivel 1956

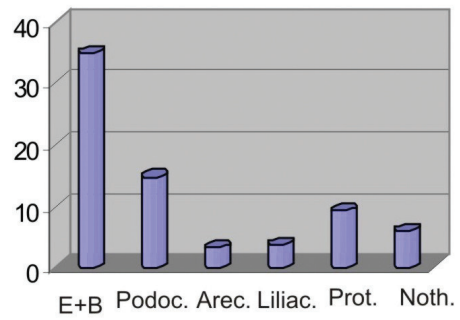

Nivel 1957

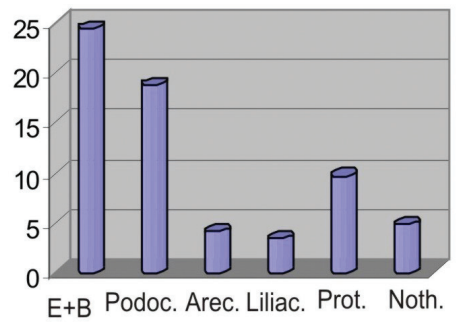

Nivel 1959

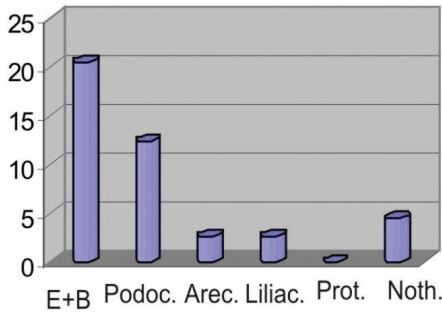

Nivel 1964

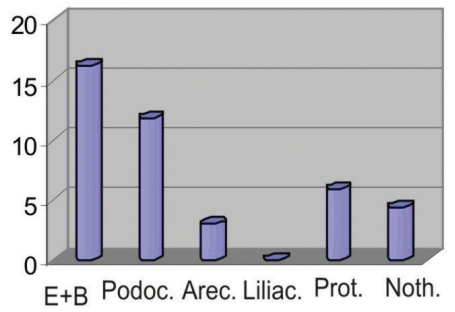

Figura 4. Diagrama de frecuencias relativas de palinomorfos continentales de la Formación Monte Chico, correspondientes a ambas zonas. Abreviaturas: E+B, esporas + briófitas; Podoc., Podocarpaceae; Arec., Arecaceae; Liliac., Liliaceae; Prot., Proteaceae; Noth., Nothafagaceae.

Figure 4. Diagram of continental palynomorphs relative frequencies of Monte Chico Formation, corresponding to both areas. Abbreviations: E+B, spores + bryophytes; Podoc., Podocarpaceae; Arec., Arecaceae; Liliac., Liliaceae; Prot., Proteaceae; Noth., Nothofagaceae.

Descripción. Grano de polen tricolporado subesférico a prolado en vista ecuatorial con extremos bien redondeados. Colpos poco distinguibles, extendidos casi hasta los polos. Poros lalongados, de pequeño tamaño, ubicados en el ecuador, de 1-2 $\mu \mathrm{m}$ de diámetro. Exina de 1,5 $\mu \mathrm{m}$ de espesor, formada por dos capas, psiladas. No se observan columelas.

Dimensiones. (3 especímenes medidos) DE 16(17)18 $\mu \mathrm{m}$. Material. MPM-MP 1960 89/6,4; MPM-MP 1946 80,5/3,2. Procedencia geográfica. Formación Monte Chico, Cuenca Austral, Provincia de Santa Cruz, Argentina.

Observaciones. Psilatricolporites sp. 1 presenta algunas similitudes con Psilatricolporites salamanquensis descripta por Archangelsky (1973), para el Paleoceno de la Formación Salamanca, Provincia del Chubut. Sin embargo, nuestros ejemplares son de menores dimensiones generales $(26 \mu \mathrm{m})$. Psilatricolporites $\mathrm{sp}$. citada para el Maastrichtiano de la Formación Loncoche, Provincia de Mendoza (Papú, 2002), tiene forma elipsoidal y la exina parece escabrada y algo más gruesa. Nuestra especie difiere de Psilatricolporites cf. P. salamanquensis (Ruiz $\&$ Quattrocchio, 1997) por sus mayores dimensiones (48 $\mu \mathrm{m}$ DP y $26 \mu \mathrm{m}$ DE) y un menor espesor de la exina (hasta $1,2 \mu \mathrm{m})$.

Afinidad botánica. Angiosperma incertae sedis. 

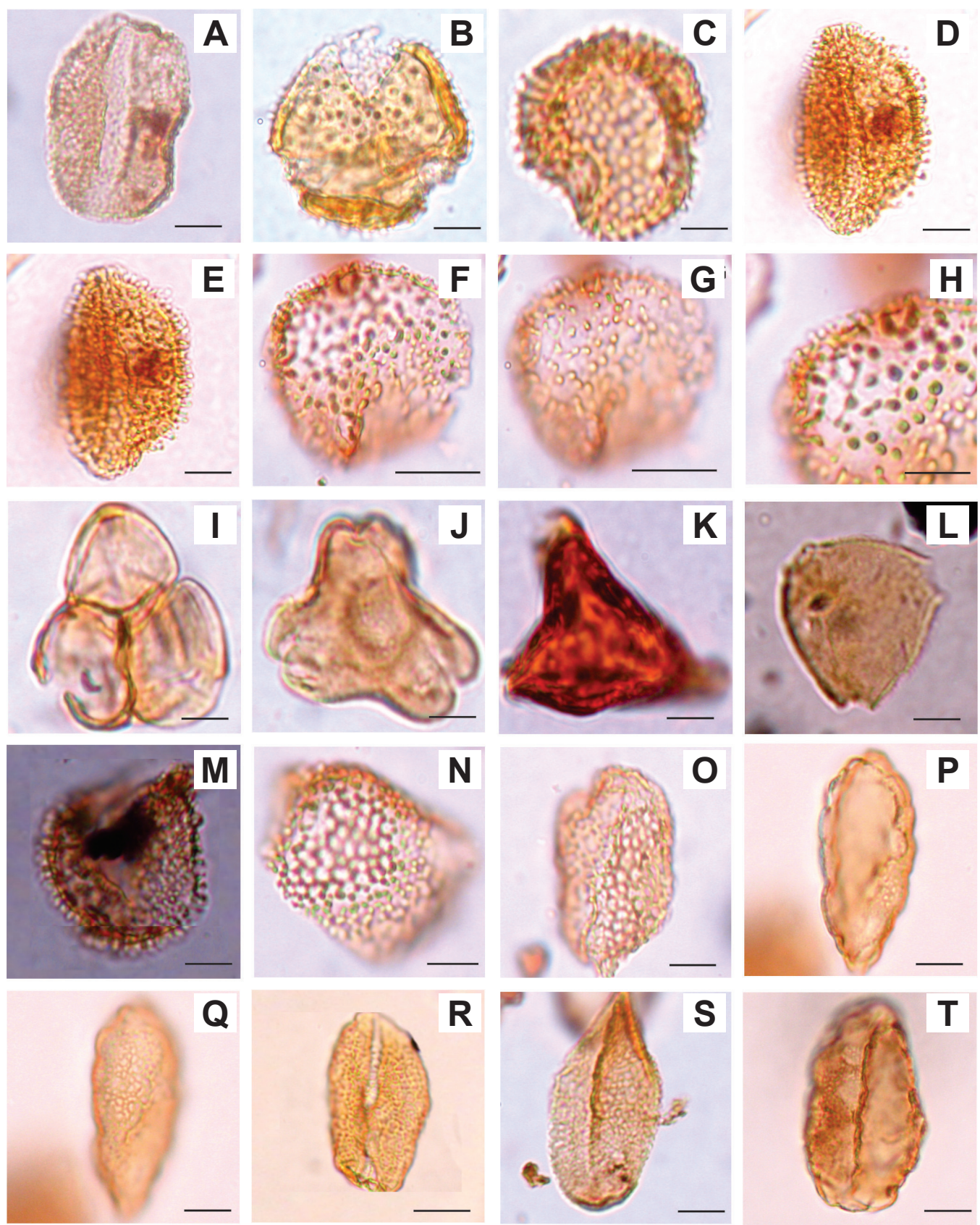

Figura 5. A, Arecipites minutiscabratus (Mclntyre, 1968) Milne, 1988, MPM-MP 1945 104/4. B, Beaupreaidites elegansiformis Cookson, 1950, MPM-MP 1951 94/9,5. C, Beaupreaidites sp. 1, MPM-MP 1955 99/15,4. D-E, Clavamonocolpites sp. 1, MPM-MP 1947 91,4/6; D, foco proximal en esculturación; E, foco proximal en la exina. F-H, Clavatricolpites sp. 1, MPM-MP 1947 91,4/6; F, foco proximal sobre el borde superior del grano; G, foco proximal en la ornamentación; H, foco mostrando detalles de las clavas. I, Ericipites scabratus Harris, 1965, MPM-MP 1948 112,7/15. J, Forcipites sp. "A" in Dettmann \& Jarzen, 1988, MPM-MP 1955 108/4,5. K, Forcipites sabulosus (Dettmann \& Playford) Dettmann \& Jarzen, 1988, MPM-MP 1951 100/6,2. L, Haloragacidites trioratus Couper, 1953, MPM-MP 1948 112,3/7,2. M, Ilexpollenites salamanquensis Archangelsky \& Zamaloa 1986, MPM-MP 1952 113,3/10,5. N, Liliacidites crassilabratus Freile, 1972, MPM-MP 1956 110,4/12,6. O, Liliacidites kaitangataensis Couper, 1953, MPM-MP 1947 103/4,8. P-Q, Liliacidites sp. cf. L. lanceolatus Stover \& Partridge, 1973, MPM-MP 1947 114,3/6,7; P, foco proximal en la exina; Q, detalle del retículo. R, Liliacidites sp. cf. L. regularis Archangelsky, 1973, MPM-MP 1948 90,7/2. S, Liliacidites variegatus Couper, 1953, MPM-MP 1946 85/2. T, Liliacidites vermireticulatus Archangelsky \& Zamaloa, 1986, MPM-MP 1947 114,3/6,7. Escalas = $10 \mu \mathrm{m}$.

Figure 5. A, Arecipites minutiscabratus (Mclntyre, 1968) Milne, 1988, MPM-MP 1945 104/4. B, Beaupreaidites elegansiformis Cookson, 1950, MPM-MP 1951 94/9,5. C, Beaupreaidites sp. 1, MPM-MP 1955 99/15,4. D-E, Clavamonocolpites sp. 1, MPM-MP 1947 91,4/6; D, proximal focus in sculpturing; E, proximal focus on the exine. F-H, Clavatricolpites sp. 1, MPM-MP 1947 91,4/6; F, proximal focus on the upper edge of the grain; G, proximal focus ornamentation; H, focus showing details of the clavas. I, Ericipites scabratus Harris, 1965, MPM-MP 1948 112,7/15. J, Forcipites sp. "A" in Dettmann \& Jarzen, 1988, MPM-MP 1955 108/4,5. K, Forcipites sabulosus (Dettmann \& Playford) Dettmann \& Jarzen, 1988, MPM-MP 1951 100/6,2. L, Haloragacidites trioratus Couper, 1953, MPM-MP 1948 112,3/7,2. M, Ilexpollenites salamanquensis Archangelsky \& Zamaloa 1986, MPM-MP 1952 113,3/10,5. N, Liliacidites crassilabratus Freile, 1972, MPM-MP 1956 110,4/12,6.0, Liliacidites kaitangataensis Couper, 1953, MPM-MP 1947 103/4,8. P-Q, Liliacidites sp. cf. L. lanceolatus Stover \& Partridge, 1973, MPM-MP 1947 114,3/6,7; P, proximal focus on exine; $\mathbf{Q}$, detail reticulum. R, Liliacidites sp. cf. L. regularis Archangelsky, 1973, MPM-MP 1948 90,7/2. S, Liliacidites variegatus Couper, 1953, MPM-MP 1946 85/2. T, Liliacidites vermireticulatus Archangelsky \& Zamaloa, 1986, MPM-MP 1947 114,3/6,7. Scale bars = $10 \mu \mathrm{m}$. 
Rhoipites Wodehouse, 1933

Especie tipo. Rhoipites bradleyi Wodehouse, 1933.

$$
\begin{gathered}
\text { Rhoipites sp.1 } \\
\text { (Figura 7F) }
\end{gathered}
$$

Descripción. Grano de polen tricolporado, subprolado, de contorno subredondeado en vista ecuatorial. Colpos relativamente largos, con membrana colpal psilada. Ora lalongados, de $3 \mu \mathrm{m}$ de diámetro. Exina gruesa, de 2,5-3 $\mu \mathrm{m}$ de espesor, semitectada, reticulada. Sexina columelada. Retículo de lúmenes subpoligonales a poligonales, disminuyendo en diámetro hacia los polos y colpos. Nexina engrosada a nivel de los poros.

Dimensiones. (3 especímenes medidos) DE 15(17,5)20 $\mu \mathrm{m}$. Material. MPM-MP 1974 94,6/6,8; 107/14,6; MPM-MP 1977 96/5,7.

Procedencia geográfica. Formación Monte Chico, Cuenca Austral, Provincia de Santa Cruz, Argentina.

Observaciones. Rhoipites romeroi Baldoni (1987), es una forma comparable, no obstante se diferencia por presentar ora circulares y mayores dimensiones generales $(30 \mu \mathrm{m})$.

Afinidad botánica. Angiosperma incertae sedis.

Subturma MONOSULCATES Iversen \& Troles-Smith, 1950

Spinizonocolpites Muller, 1968

Especie tipo. Spinizonocolpites echinatus Muller, 1968.

\section{Spinizonocolpites $\mathrm{sp} .1$}

(Figura 7L)

Descripción. Grano de polen monocolpado de contorno elíptico a subesferoidal. Colpo situado ecuatorialmente, apenas visible, dividiendo al grano en dos mitades desiguales. Exina fina de $1 \mu \mathrm{m}$ de espesor, superficialmente lisa a escabrada; ornamentada con abundantes espinas cónicas, de 2-3 $\mu \mathrm{m}$ de largo, de extremos puntiagudos, distribuídas en forma irregular sobre la superficie del grano.

Dimensiones. (3 especímenes medidos) DE 17(18)19 $\mu \mathrm{m}$; DP 30(31) $32 \mu \mathrm{m}$.

Material. MPM-MP 1975 110/8; MPM-MP 1971 116,4/10,3. Procedencia geográfica. Formación Monte Chico, Cuenca Austral, Provincia de Santa Cruz, Argentina.

Observaciones. Spinizonocolpites sp. 1 difiere de Spinizonocolpites hialinus (Archangelsky \& Zamaloa, 1986) descripta para la Formación Bororó (Paleoceno) de la Provincia del Chubut (Archangelsky, 1973) porque presenta una forma subesferoidal más alargada, exina más delgada y los elementos esculturales son de menores dimensiones con una disposición más irregular.

Afinidad botánica. Familia Palmae.

Subturma TRIPTICHES Naumova, 1939

Tricolpites Cookson ex Couper, 1953

Especie tipo. Tricolpites reticulatus Cookson, 1947.
Tricolpites $\mathrm{sp} .1$

(Figura 7Q)

Descripción. Grano de polen tricolpado, de contorno subesferoidal, prolato en vista ecuatorial, trilobulado en vista polar, semitectado. Colpos largos y profundos. Exina gruesa de $2 \mu \mathrm{m}$ de espesor, reticulada; retículo muy fino y apretado, de muros bajos y relativamente gruesos, de $0,7-0,8 \mu \mathrm{m}$ de ancho y lúmenes subcirculares, de hasta $1 \mu \mathrm{m}$ de diámetro en los mesocolpios, disminuyendo hacia los polos.

Dimensiones. (2 especímenes medidos) DE 20-32 $\mu \mathrm{m}$.

Material. MPM-MP 1965 104/6; MPM-MP 1951 90,5/3,7.

Procedencia geográfica. Formación Monte Chico, Cuenca Austral, Provincia de Santa Cruz, Argentina.

Observaciones. Tricolpites sp. 1 se distingue de Tricolpites sp. 2 por presentar un retículo con lúmenes de mayor diámetro y muros más anchos y lúmenes de un tamaño; las dimensiones de ambas son comparables. Tricolpites cf. reticulatus (Cookson, 1947) registrada en la Formación Monte Chico, presenta un rango de dimensiones ligeramente menor, una forma esferoidal, una exina más fina y lúmenes del retículo más chicos. Tricolpites reticulatus (Cookson, 1947) registrada en la Formación Cerro Cazador (Povilauskas, 2010), se caracteriza por presentar una forma más esferoidal, exina más fina y lúmenes del retículo de menor tamaño.

Afinidad botánica. Familia Gunneraceae.

Tricolpites sp. 2

(Figura 7R)

Descripción. Grano de polen tricolpado, de forma esferoidal, oblato en vista ecuatorial, contorno subtriangular a subredondeado en vista polar, lados convexos y bordes redondeados. Colpos largos, de $7 \mu \mathrm{m}$ de profundidad. Exina formada por dos capas, de espesor uniforme (1,5-2 $\mu \mathrm{m})$, microreticulada. En los márgenes colpoides la exina se afina ligeramente $(1 \mu \mathrm{m}$ de espesor). Microretículo con lúminas menores a $0,4 \mu \mathrm{m}$ de diámetro, desarrollado de manera uniforme sobre la exina. Muros delgados $\mathrm{y}$ angostos, de menos de $0,5 \mu \mathrm{m}$ de ancho.

Dimensiones. (2 especímenes medidos) DE 25-27,5 $\mu \mathrm{m}$.

Material. MPM-MP 1945 110/3,2.

Procedencia geográfica. Formación Monte Chico, Cuenca Austral, Provincia de Santa Cruz, Argentina.

Observaciones. La especie aquí descripta presenta características similares a Tricolpites reticulatus (Cookson, 1947), difiriendo en el microretículo; el cual es más chico en los especímenes que aquí se presentan y la forma general del grano es subredondeada. Ambas especies coinciden en tamaño. Afinidad botánica. Familia Gunneraceae. Subturma PTYCHOTRIPORINES Naumova, 1939
Infraturma PROLATI Erdtman, 1943

Tricolporites Cookson, 1947

Especie tipo. Tricolporites sphaerica Cookson, 1947.

Tricolporites sp. 1

(Figura 7S) 

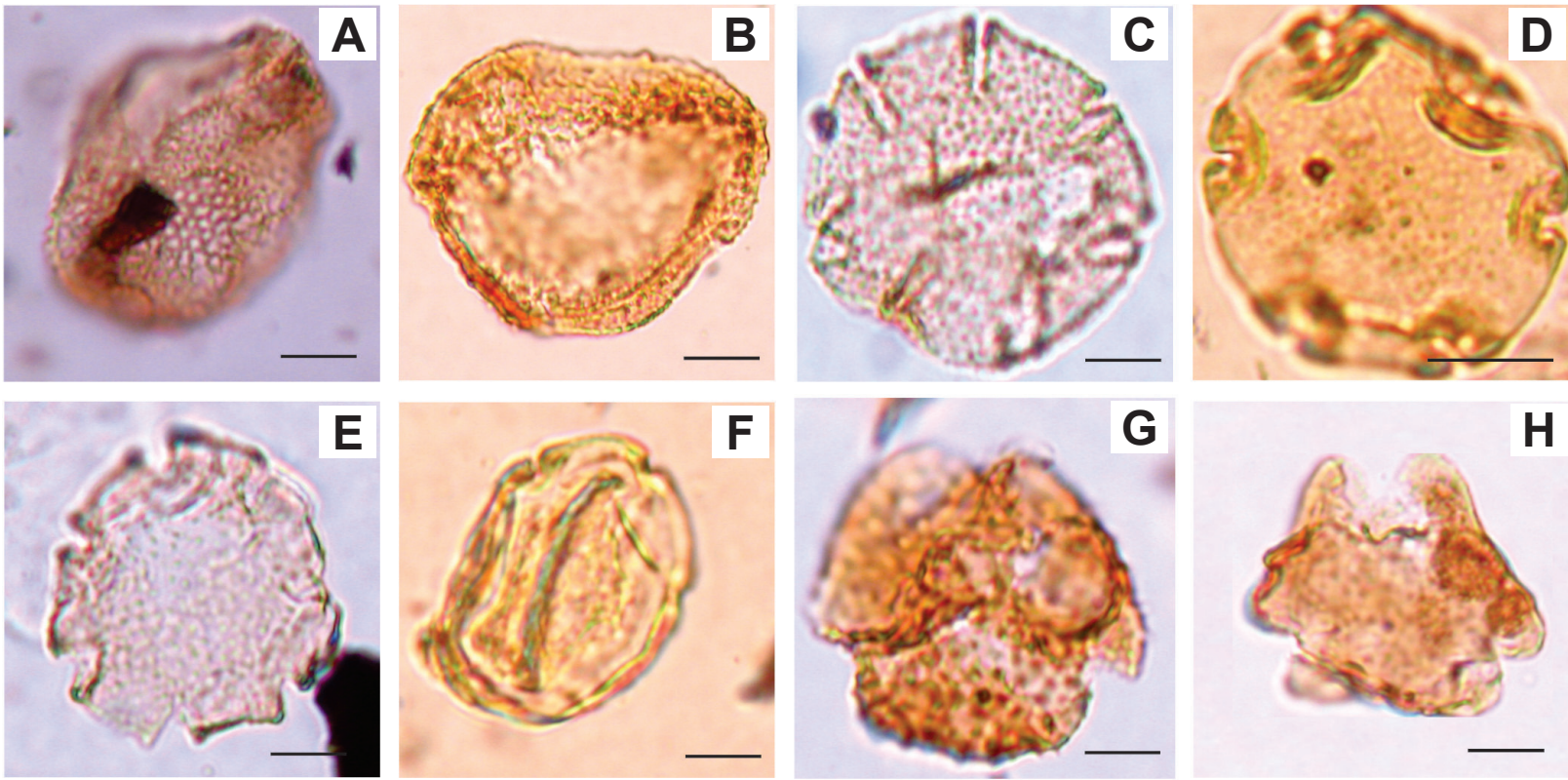

G

H
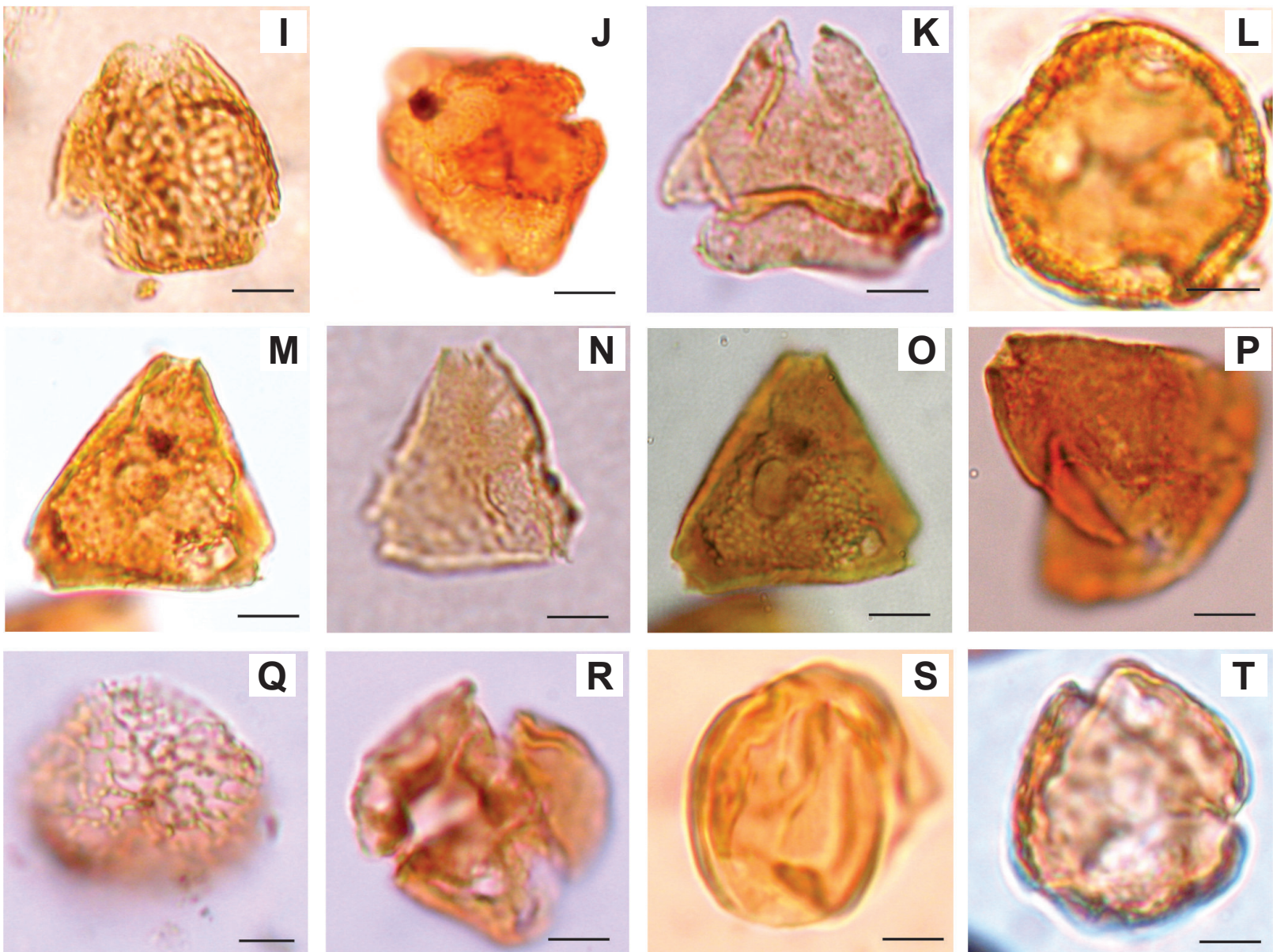

Figura 6/Figure 6. A, Liliacidites sp. 1, MPM-MP 1949 102,1/14,5. B, Longapertites patagonicus Archangelsky, 1973, MPM-MP 1960 104/13. C Nothofagidites dorotensis Romero, 1973, MPM-MP 1955 111/3,5. D, Nothofagidites kaitangataensis (Te Punga) Romero, 1973, MPM-MP 1957 101/7. E, Nothofagidites nana Romero, 1977, MPM-MP 1958 95,3/8. F, Nothofagidites saraensis Menéndez \& Caccavari de Fílice, 1975, MPMMP 1958 119,3/9,6. G, Peninsulapollis askiniae Dettmann \& Jarzen, 1988, MPM-MP 1951 107,5/21,4. H, Peninsulapollis gillii (Cookson, 1957) emend. Dettmann \& Jarzen, 1988, MPM-MP 1947 114/15. I, Peninsulapollis truswellidae Dettmann \& Jarzen, 1988, MPM-MP 1945 112,1/5. J, Peninsulapollis sp. cf. P. truswellidae Dettmann \& Jarzen, 1988, MPM-MP 1946 90/8,5. K, Peninsulapollis sp. 1, MPM-MP 1947 100/20,2. L, Periporopollenites demarcatus (Stover) Stover \& Partridge, 1973, MPM-MP 1977 99/16,4. M, Proteacidites beddoesii Stover\& Partridge, 1973, MPM-MP 1945 107/11. N, Proteacidites parvus Cookson, 1950, MPM-MP 1970 93,1/4. O, Proteacidites subscabratus Couper, 1960, MPM-MP 1945 103/1,8. P, Proteacidites tenuiexinus Stover in Stover \& Partridge, 1973, MPM-MP 1947 91,4/6,7. Q, Pseudowinterapollis couperi Krutzsch, 1970 emend. Mildenhall, 1979, MPM-MP 1952 101,3/8. R-S, Psilatricolpitespatagonicus Freile, 1972; R, MPM-MP 1945 100,5/11,2 (en vista polar); S, MPM-MP 1953 100/5 (en vista ecuatorial / equatorial view). T, Psilatricolpites sp. 1, MPM-MP 1946 37/95,6. Escalas $=10 \mu \mathrm{m} / \mathrm{Scale}$ bars $=10 \mu \mathrm{m}$. 
Descripción. Grano de polen tricolporado, isopolar, prolato a subprolato. Colpos largos, extendiéndose hasta los polos, y apocolpios pequeños. Poros de 3-5 $\mu \mathrm{m}$ diámetro, en ocasiones no se visualizan bien por la esculturación densa de la pared. Exina de 1,5-1,8 $\mu \mathrm{m}$ de espesor, uniforme a lo largo del grano. Nexina y sexina de igual grosor. Esculturación formada por clavas de $2 \mu \mathrm{m}$ de alto y $0,8-1 \mu \mathrm{m}$ de ancho, dispuestas densamente sobre la superficie del grano.

Dimensiones. (2 especímenes medidos) DE $20 \mu \mathrm{m}$; DP $38 \mu \mathrm{m}$. Material. MPM-MP 1947 114,3/5; 89/9,5.

Procedencia geográfica. Formación Monte Chico, Cuenca Austral, Provincia de Santa Cruz, Argentina.

Observaciones. Esta especie se halló en forma escasa en algunos niveles; y se comparó con la especie Tricolporites sp. 1 descripta por Freile (1972) para el Daniano de la Formación Cerro Dorotea, que se diferencia por presentar una exina algo más gruesa y una ornamentación más grosera. Tricolporites sp. 2 descripta para la misma Formación (Freile, 1972), también presenta diferencias en la esculturación y es de menores dimensiones generales.

Afinidad botánica. Angiosperma incertae sedis.

\section{DISCUSIÓN}

Las asociaciones palinológicas de la Formación Monte Chico están dominadas por elementos continentales que alcanzan entre el 50-88 \% del espectro palinológico (Tabla 1). Los granos de polen pertenecientes a las angiospermas se encuentran en porcentajes variables que oscilan entre 1144,6\% (Tabla 2), representados principalmente por Arecaceae (Arecipites minutiscabratus, Longapertites patagonicus), Proteaceae (Forcipites spp., Triatriopollenites bertelsii, T. lateflexus) y Liliaceae (Liliacidites spp.); y en forma subordinada Gunneraceae (Tricolpites reticulatus) (Figura 4); que evidencian el carácter marino marginal de la cuenca en el sector de estudio, al SO de la Provincia de Santa Cruz.

Entre los elementos marinos hallados se reconocen dinoquistes de dinoflagelados (10-51\%) y acritarcos (0,32-1\%) (Tabla 1; Figuras 3, 4). La presencia de microplancton marino más la gran diversidad de esporas de briófitas y pteridófitas, y granos de polen de gimnospermas, que han sido motivo de estudio en trabajos anteriores (Povilauskas, 2011, 2012), sumado a la gran diversidad de granos de polen de angiospermas (este trabajo), permite sugerir condiciones marino marginales para la formación con la presencia de cuerpos de agua dulce cercanos al medio de depositación (Papú \& Sepúlveda, 1995), y el desarrollo de comunidades vegetales dominadas por Arecaceae y Proteaceae con una tupida cubierta vegetal de helechos bajo condiciones de clima cálido y húmedo. Por su parte, la presencia de Sparganiaceapollenites barungensis (Sparganiaceae) indicaría la presencia de suelos inundados o muy húmedos.

Por otra parte, las Arecaceae reúnen plantas arbustivas y arborescentes distribuidas en la actualidad en las regiones ecuatorial, tropical y subtropical. Las bajas frecuencias de Araucariaceae y Nothofagaceae observadas a lo largo de toda la unidad, parecen sugerir que los bosques se habrían desarrollado en altos topográficos a cierta distancia de la cuenca de depositación. La ausencia de granos de polen proveniente de plantas xerófilas, como las Efedreceae, confirma la buena disponibilidad de agua en las áreas circundantes.

Como resultado, se interpreta que toda la unidad se habría depositado bajo un régimen costero marginal, probablemente somero, bajo condiciones de clima cálido y al menos localmente húmedo.

\section{AGRADECIMIENTOS}

La autora agradece a M. Arai y a L. Antonioli por los comentarios críticos que permitieron mejorar el manuscrito original; a V. Barreda por sus detallados comentarios; a O. Cárdenas por la ayuda brindada en el procesamiento de las muestras palinológicas. Este trabajo fue realizado bajo el apoyo económico brindado por el CONICET y a la Agencia Nacional de Promoción Científica y Tecnológica (PICT 32320).

\section{REFERENCIAS}

Archangelsky, S. 1973. Palinología del Paleoceno de Chubut 1. Descripciones sistemáticas. Ameghiniana, 10:339-399.

Archangelsky, S. \& Zamaloa, M.C. 1986. Nuevas descripciones palinológicas de las formaciones Salamanca y Bororó, Paleoceno de Chubut, República Argentina. Ameghiniana, 23:35-46.

Baldoni, A.M. 1987. Estudios palinológicos de la zona de Collón Cura, Provincia del Neuquén, Sobre elementos del Terciario Inferior y redepositados del Cretácico Inferior. Revista Española de Micropaleontología, 19:367-411.

Cookson, I.C. 1947. Plant microfossils from the lignites of the Kerguelen Archipelago. British and New Zealand Antarctic Research Expedition, 1929-1931, 2:129-142 (Reports, Series A).

Cookson, I.C. 1950. Fossil pollen grains of Proteaceous type from Tertiary deposits in Australia. Australian Journal of Science, 3:166-177.

Cookson, I.C. 1957. On some Australian Tertiary spores and pollen grains that extend the geological and geographical distribution of living genera. Proceedings of the Royal Society of Victoria, 69:41-53.

Couper, R.A. 1953. Upper Mesozoic and Cainozoic spores and pollen grains from New Zealand. Bulletin of the New Zealand Geological Survey, Paleontology, 22:1-77.

Couper, R.A. 1960. New Zealand Mesozoic and Cainozoic plant microfossils. Bulletin of the New Zealand Geological Survey, Paleontology, 32:1-87.

Dettmann, M.E. \& Jarzen, D.M. 1988. Angiosperm pollen from uppermost Cretaceous strata of southeastern Australia and the Antarctic Peninsula. Memoir of the Association of Australasian Palaeontologists, 5:217-237.

Freile, C. 1972. Estudio palinológico de la Formación Cerro Dorotea (Maastrichtiano-Paleoceno) de la provincia de Santa Cruz. Revista de Museo de La Plata, 6:39-63.

Harris, W.K. 1965. Basal Tertiary microfloras from the Princetown area, Victoria, Australia. Palaeontographica, Abteilung B, 115:75-106.

Harris, W.K. 1972. New form species of pollen from southern Australian early Tertiary sediments. Transactions of the Royal Society of Australia, 96:53-65. 

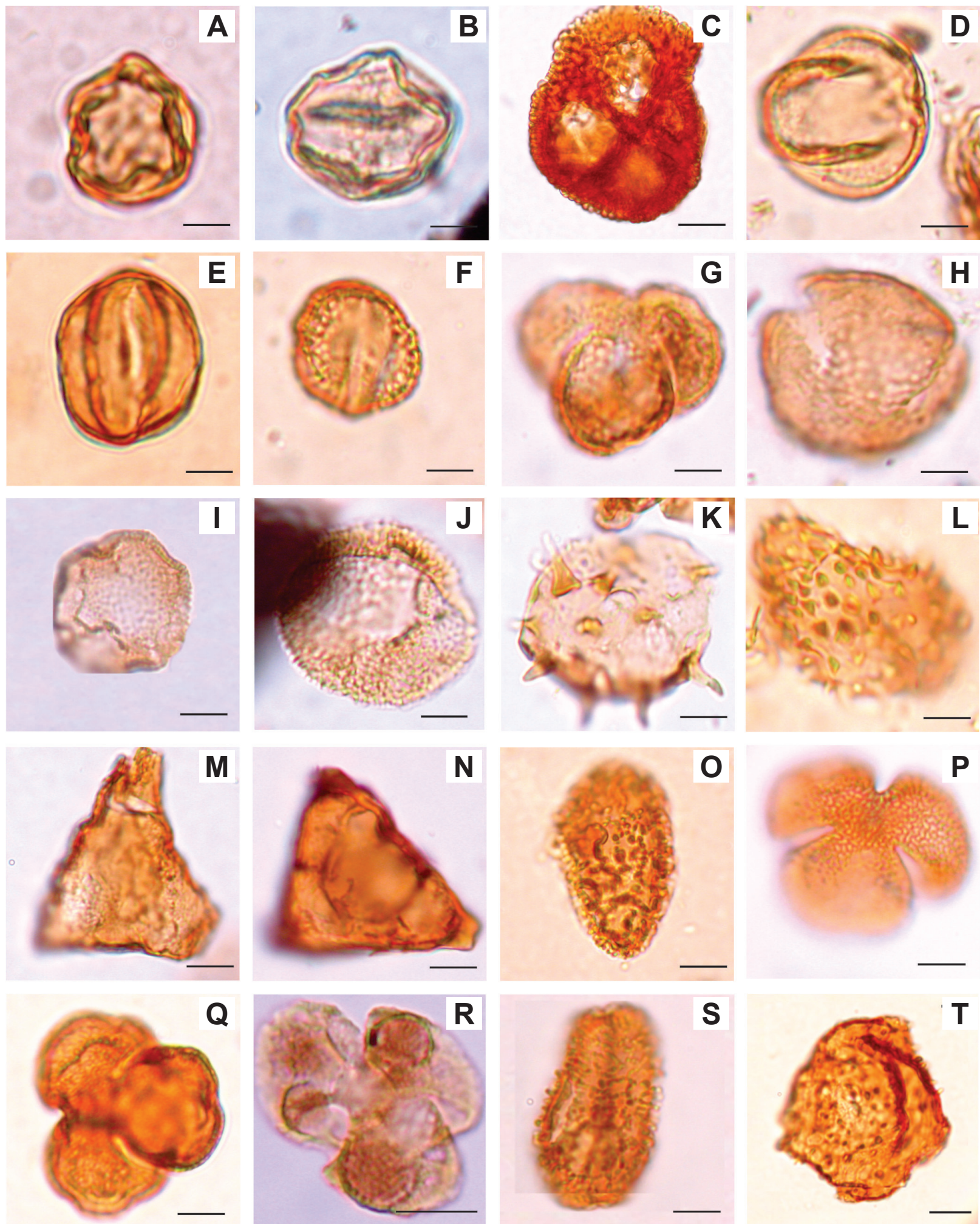

Figura/Figure 7. A, Psilatricolporites sp. cf. P. salamanquensis Archangelsky \& Zamaloa, 1986, MPM-MP 1945 114,4/2,8. B, Psilatricolporites sp. 1, MPM-MP 1960 89/6,4. C, Quadraplanus brossus Stover in Stover \& Partridge, 1973, MPM-MP 1949 34,6/92,5. D, Rhoipites baculatus Archangelsky, 1973, MPM-MP 1978 109,8/2,3. E, Rhoipites minusculus Archangelsky, 1973, MPM-MP 1945 103/1,8. F, Rhoipites sp. 1, MPMMP 1974 94,6/6,8. G, Rousea microreticulata Archangelsky \& Zamaloa, 1986, MPM-MP 1945 117,7/8. H, Rousea patagonica Archangelsky, 1973, MPM-MP 1957 99,3/11. I, Senipites tercrassata Archangelsky, 1973, MPM-MP 1945 96/18. J, Sparganiaceapollenites barungensis Harris, 1972, MPM-MP 1946 116/4,3. K, Spinizonocolpites hialinus Archangelsky \& Zamaloa, 1986, MPM-MP 1945 97,2/4,6. L, Spinizonocolpites sp. 1, MPM-MP 1975 110/8. M, Triatriopollenites bertelsii Archangelsky, 1973, MPM-MP 1945 107,5/2. N, Triatriopollenites lateflexus Archangelsky, 1973, MPM-MP 1949 113,5/3. O, Tricolpites bibaculatus Archangelsky \& Zamaloa, 1986, MPM-MP 1948 106,4/10,8. P, Tricolpites reticulatus Cookson, 1947, MPM-MP 1948 98,5/10,6. Q, Tricolpites sp. 1, MPM-MP 1951 90,5/3,7. R, Tricolpites sp. 2, MPM-MP 1945 110/3,2. S, Tricolporites sp. 1, MPM-MP 1947 114,3/5. T, Triporopollenites sp. cf. T. ambiguus (Stover) Stover \& Partridge, 1973, MPM-MP 1946 105,7/4. Escala $=10 \mu \mathrm{m}$. Scale bars $=10 \mu \mathrm{m}$. 
Tabla 1. Frecuencias relativas (palinomorfos marinos/continentales) en las dos zonas de estudio.

Table 1. Relative frequencies (palynomorphs marine/continental) in the two study areas.

\begin{tabular}{lccccccccccc}
\hline Origen (\%) & 1945 & 1947 & 1949 & 1952 & 1953 & 1954 & 1955 & 1956 & 1957 & 1959 & 1964 \\
Continental & 87,4 & 88,1 & 88 & 66,9 & 58,2 & 66,9 & 85,9 & 82,1 & 80,4 & 50,5 & 48,2 \\
Marino & 12,6 & 11,9 & 11 & 33,1 & 41,8 & 33 & 14,1 & 17,9 & 19,6 & 49,5 & 51,8 \\
\hline
\end{tabular}

Tabla 2. Abundancia relativa de los palinomorfos en los niveles seleccionados de la Formación Monte Chico, correspondientes a ambas zonas.

Table 2. Relative abundance of palynomorphs in the selected levels of Formation Monte Chico, for both areas.

\begin{tabular}{|c|c|c|c|c|c|c|c|}
\hline Niveles & Esporas+Briófitas & Podocarpaceae & Arecáceas & Liliáceas & Proteaceae & Nothofagaceae & Angiospermas indet. \\
\hline 1945 & 41,3 & 16,3 & 7,8 & 6 & 8,4 & 0 & 20,2 \\
\hline 1947 & 36 & 13,4 & 3,7 & 9,5 & 19,4 & 0 & 18 \\
\hline 1949 & 42,8 & 22,2 & 3,9 & 8,1 & 8,4 & 0 & 14,6 \\
\hline 1952 & 53,4 & 24,4 & 0 & 6,3 & 8,7 & 0 & 7,2 \\
\hline 1953 & 51,7 & 14,1 & 2,2 & 4,3 & 10,3 & 0,8 & 16,6 \\
\hline 1954 & 48,7 & 20,6 & 10,4 & 1,5 & 0 & 7,4 & 11,4 \\
\hline 1955 & 35 & 24 & 3,3 & 0 & 14,7 & 8,9 & 14,1 \\
\hline 1956 & 40,9 & 17,3 & 4,4 & 4,5 & 8,3 & 7,1 & 17,5 \\
\hline 1957 & 30,3 & 23,5 & 5,8 & 4,1 & 9,2 & 5,8 & 21,3 \\
\hline 1959 & 40,8 & 26,9 & 4,9 & 4,9 & 0 & 8,5 & 14 \\
\hline 1964 & 32,7 & 23,8 & 6 & 0 & 10,3 & 8,9 & 18,3 \\
\hline
\end{tabular}

Jaramillo, C.A. \& Dilcher, D.L. 2001. Middle Paleocene palynology of Central Colombia, South America: a study of pollen and spores from tropical latitudes. Palaeontographica, Abteilung B, 258:87-213.

Krutzsch, W. 1970. Atlas der mittel- und jungtertiaren dispersen Sporen- und Pollen-sowie der Mikroplanktonformen des nordlichen Mitteleuropas. Lieferung 7: Monoporate, monocolpate, longicolpate, dicolpate und ephedroide (polyplicate) Pollenformen. Berlin, Veb Gustav Fischer Verlag Jena, 175 p.

Mc Intyre, D. J. 1968. Futher new pollen species from New Zealand Tertiary and uppermost Cretaceous deposits. New Zealand Journal of Botany, 6:177-204.

Menéndez, C. \& Caccavari, M.A. 1975. Las especies de Nothofagidites (polen fósil de Nothofagus) de sedimentos Terciarios y Cretácicos de Estancia La Sara, Norte de Tierra del Fuego, Argentina. Ameghiniana, 12:165-183.

Mildenhall, D.C. 1979. Palynology of the Waipipian and Hautawan Stages (Pliocene and Pleistocene), Wanganui, New Zealand (note). New Zealand Journal of Geology and Geophysics, 21:775-777.

Milne, L.A., 1988. Palynology of a late Eocene lignitic sequence from the western margin of the Eucla Basin, Western Australia. Memoir of the Association of Australasian Palaeontologists, 5:285-310.

Müller, J. 1968. Palynology of the Pedawan and Plateau Sandstone formations (Cretaceous-Eocene) in Sarawak, Malaysia. Micropaleontology, 14:1-37.

Müller, J.; Di Giacomo, E. \& Van Erve, A. 1987. A palynologic zonation for the Cretaceous, Tertiary and Quaternary of Northern South America. AASP Contributions Series, 19:7-79.

Papú, O.H. 2002. Nueva microflora de edad Maastrichtiana en la localidad de Calmu-Co, sur de Mendoza, Argentina. Ameghiniana, 39:415-426.

Papú, O.H. \& Sepúlveda, E.G. 1995. Datos palinológicos de la Formación Los Alamitos en la localidad de Montoniló, Departamento 25 de Mayo, Río Negro, Argentina. Sus relaciones con unidades colindantes coetáneas. In: CONGRESO ARGENTINO DE PALEONTOLOGÍA Y BIOESTRATIGRAFÍA, 6, 1995. Actas, Trelew, p. 195-200.

Pierce, R.L. 1961. Lower Upper Cretaceous plant microfossils from Minnesota. Minnesota Geological Survey Bulletin, 42:1-86.
Pocock, S.A. 1962. Microfloral analysis and age determinations of strata at the Jurassic-Cretaceous boundary in the western Canada Plains. Palaeontographica, Abteilung B, 111:1-95.

Povilauskas, L. 2010. Palinología del entorno Cretácico/Paleógeno del SO de la Provincia de Santa Cruz: significado estratigráfico y paleoambiental. Universidad Nacional de La Plata, Tesis Doctoral. http://sedici.unlp.edu.ar?id=ARG-UNLP-TPG-0000001067.

Povilauskas, L. 2011. Palinología de la Formación Monte Chico (Cretácico Tardío) de la Provincia de Santa Cruz, Argentina: Esporas. Revista Brasileira de Paleontología, 14:255-268. doi: 10.4072/rbp.2011.3.05

Povilauskas, L. 2012. Palinología de la Formación Monte Chico (Cretácico Tardío) de la Provincia de Santa Cruz, Argentina: Granos de polen de Gimnospermas. Revista Brasileira de Paleontología, 15:85-94. doi: 10.4072/rbp.2012.1.07

Romero, E.J. 1973. Polen fósil de Nothofagus (Nothofagidites) del Cretácico y Paleoceno de Patagonia. Revista del Museo de La Plata, 7:291-303.

Romero, E.J. 1977. Polen de gimnospermas y fagáceas de la Formación Río Turbio (Eoceno), Santa Cruz, Argentina. Buenos Aires, Fundación para la Educación, la Ciencia y la Cultura, 223 p.

Ruiz, L.C. \& Quattrocchio, M.E. 1997. Estudio palinológico de la Formación Pedro Luro (?Maastrichtiano-Paleoceno) en la Cuenca del Colorado, Republica Argentina. Parte 1: Esporas triletes, Laevigati, Murornati, Tricassati, Cingulati y Zonati. Revista Española de Micropaleontología, 29:13-29.

Stover, L.E. \& Partridge, A.D. 1973. Tertiary and Late Cretaceous spores and pollen from the Gippsland Basin, southeastern Australia. Proceedings of the Royal Society of Victoria, 85:237286.

van der Hammen, T. 1956. Nomenclatura palinológica sistemática. Boletín Geológico, 4:23-62.

van der Hammen, T. \& Wijmstra, T. A. 1964. A palynological study on the Tertiary and Upper Cretaceous of British Guiana. Leidse Geologische Mededelingen, 30:183-241.

Wodehouse, R.P. 1933. The oil shales of the Eocene Green River Formation. Bulletin of the Torrey Botanical Club, 60:479-535.

Received in January, 2011; accepted in December, 2012. 
Apéndice 1. Lista de especies identificadas.

Appendix 1. List of species identified.

\section{Angiospermas}

Arecipites minutiscabratus (McIntyre, 1968) Milne, 1988 (Figura 5A)

Beaupreaidites elegansiformis Cookson, 1950 (Figura 5B)

Beaupreaidites sp. 1 (Figura 5C)

Clavamonocolpites sp. 1 (Figuras 5D-E)

Clavatricolpites sp. 1 (Figuras 5F-H)

Ericipites scabratus Harris, 1965 (Figura 5I)

Forcipites sp. "A" en Dettmann \& Jarzen 1988 (Figura 5J)

Forcipites sabulosus (Dettmann \& Jarzen, 1988 (Figura 5K)

Haloragacidites trioratus Couper, 1953 (Figura 5L)

Ilexpollenites salamanquensis Archangelsky \& Zamaloa, 1986 (Figura 5M)

Liliacidites crassilabratus Freile, 1972 (Figura 5N)

Liliacidites kaitangataensis Couper, 1953 (Figura 5O)

Liliacidites sp. cf. L. lanceolatus Stover \& Partridge, 1973 (Figuras 5P-Q)

Liliacidites sp. cf. L. regularis Archangelsky, 1973 (Figura 5R)

Liliacidites variegatus Couper, 1953 (Figura 5S)

Liliacidites vermireticulatus Archangelsky \& Zamaloa, 1986 (Figura 5T)

Liliacidites sp. 1 (Figura 6A)

Longapertites patagonicus Archangelsky, 1973 (Figura 6B)

Nothofagidites dorotensis Romero, 1973 (Figura 6C)

Nothofagidites kaitangataensis (Te Punga) Romero, 1973 (Figura 6D)

Nothofagidites nana Romero, 1977 (Figura 6E)

Nothofagidites saraensis Menéndez \& Caccavari, 1975 (Figura 6F)

Peninsulapollis askiniae Dettmann \& Jarzen, 1988 (Figura 6G)

Peninsulapollis gillii (Cookson, 1957) emend. Dettmann \& Jarzen, 1988 (Figura 6H)

Peninsulapollis truswellidae Dettmann \& Jarzen, 1988 (Figura 6I)

Peninsulapollis sp. cf. P. truswellidae Dettmann \& Jarzen, 1988 (Figura 6J)

Peninsulapollis sp. 1 (Figura 6K)

Periporopollenites demarcatus Stover \& Partridge, 1973 (Figura 6L)

Proteacidites beddoesii Stover \& Partridge, 1973 (Figura 6M)

Proteacidites parvus Cookson, 1950 (Figura 6N)

Proteacidites subscabratus Couper, 1960 (Figura 6O)

Proteacidites tenuiexinus Stover in Stover \& Partridge, 1973 (Figura 6P)

Pseudowinterapollis couperi Krutzsch, 1970 emend. Mildenhall, 1979 (Figura 6Q)

Psilatricolpites patagonicus Freile, 1972 (Figuras 6R-S)

Psilatricolpites sp. 1 (Figura 6T)

Psilatricolporites sp. cf. P. salamanquensis Archangelsky \& Zamaloa, 1986 (Figura 7A)

Psilatricolporites sp. 1 (Figura 7B)

Quadraplanus brossus Stover in Stover \& Partridge, 1973 (Figura 7C)

Rhoipites baculatus Archangelsky, 1973 (Figura 7D)

Rhoipites minusculus Archangelsky, 1973 (Figura 7E)

Rhoipites sp. 1 (Figura 7F)

Rousea microreticulata Archangelsky \& Zamaloa, 1986 (Figura 7G)

Rousea patagonica Archangelsky, 1973 (Figura 7H)

Senipites tercrassata Archangelsky, 1973 (Figura 7I)

Sparganiaceapollenites barungensis Harris, 1972 (Figura 7J)

Spinizonocolpites hialinus Archangelsky \& Zamaloa, 1986 (Figura 7K)

Spinizonocolpites sp. 1 (Figura 7L)

Triatriopollenites bertelsii Archangelsky, 1973 (Figura 7M)

Triatriopollenites lateflexus Archangelsky, 1973 (Figura 7N)

Tricolpites bibaculatus Archangelsky \& Zamaloa, 1986 (Figura 7O)

Tricolpites reticulatus Cookson, 1947 (Figura 7P)

Tricolpites sp. 1 (Figura 7Q)

Tricolpites sp. 2 (Figura 7R)

Tricolporites sp. 1 (Figura 7S)

Triporopollenites sp. cf. T. ambiguus (Stover) Stover \& Partridge, 1973 (Figura 7T) 